\title{
Effects of Aqueous-Supplementing Artificial Tears in Wearers of Biweekly Replacement Contact Lenses vs Wearers of Daily Disposable Contact Lenses
}

This article was published in the following Dove Press journal:

Clinical Optometry

\author{
Silvia Tavazzi $\mathbb{D}^{1,2}$ \\ Riccardo Origgi' \\ Martina Anselmi (D) \\ Andrea Corvino (iD) \\ Sara Colciago' \\ Matteo Fagnola ${ }^{2}$ \\ Silvia Bracco' \\ Fabrizio Zeri $\mathbb{D}^{1-3}$ \\ 'Department of Materials Science, \\ University of Milano-Bicocca, Milan, Italy; \\ ${ }^{2}$ Research Centre in Optics and \\ Optometry (COMiB), University of \\ Milano-Bicocca, Milan, Italy; ${ }^{3}$ Ophthalmic \\ Research Group, School of Life and \\ Health Sciences, Aston University, \\ Birmingham, UK
}

Purpose: To compare the effects of artificial tears (ATs) in wearers of biweekly replacement silicone hydrogel contact lenses (BW-Ws) and wearers of daily disposable contact lenses (DD-Ws) of the same material.

Materials and Methods: The aqueous-supplementing ATs, OPTOyalA and OPTOidro, were assigned to be used for 2 weeks to healthy and young subjects: 1) 20 (8 and 12, respectively) BW-Ws wearing silicone hydrogel somofilcon A CLs (Clariti Elite), 2) 18 (9 and 9, respectively) DD-Ws wearing silicone hydrogel somofilcon A CLs (Clarity 1 Day), and 3 ) a control group of 33 (16 and 17, respectively) N-Ws. Ocular symptoms and comfort, tear volume and stability, and ocular surface condition were assessed by Ocular Surface Disease Index (OSDI), 5-Item Dry Eye Questionnaire (DEQ5), tear meniscus height (TMH), non-invasive tear break-up time (NIBUT), and evaluation of ocular redness (OR). The assessment was performed before and after 15 days of use of the ATs in the 3 groups (BWWs, DD-Ws, and N-Ws).

Results: No clear significant difference was noted in symptoms and signs between OPTOyalA and OPTOidro irrespectively of the group of people studied. ATs use for 15 days produced a significant improvement in DEQ5 and OR in DD-Ws $(\Delta=-34 \%$, $\mathrm{p}=0.006 ; \Delta=-23 \%, \mathrm{p}<0.001)$ and in N-Ws $(\Delta=-21 \%, \mathrm{p}=0.001 ; \Delta=-10 \%, \mathrm{p}=0.006)$ but not in BW-Ws $(\Delta=-5 \%, \mathrm{p}=0.072 ; \Delta=-2 \%, \mathrm{p}=0.257)$. No significant change was noted for TMH. Conclusion: In young and healthy subjects, the aqueous-supplementing effect of the ATs under consideration is more a rinsing and tear replacem ent effect than an increase in tear volume, and it produces an improvement of the eye redness and ocular symptoms. Contact lens wear influenced the effectiveness of ATs in a way which is correlated with the CL replacement schedule.

Keywords: artificial tears, contact lenses, somofilcon A

\section{Introduction}

In the first approximation, the tear film is typically described by a mixed inner aqueous-mucins layer with an outer lipid layer. ${ }^{1}$ A stable and healthy tear film provides a protective layer, which guarantees good eye comfort and vision thanks to the hydration and nutrition of the cornea and conjunctiva, the protection of the ocular surface from dust, dirt particles, and foreign bodies, and the maintenance of corneal transparency. ${ }^{1-3}$ When the quality or quantity of tears are compromised due
Correspondence: Silvia Tavazzi Email silvia.tavazzi@unimib.it 
to some dysfunctions, tear film instability and dry eye symptoms may occur. ${ }^{4-9}$ It has been indicated that the prevalence of dry eye ranges from approximately 5\% to $50 \%$ when the diagnosis is based on symptoms with or without signs. $^{8}$ However, when the diagnosis is based primarily on signs, studies generally reported higher and more variable rates of disease, up to $75 \%{ }^{8}$

Artificial tears (ATs) are tear substitutes, which are available over-the-counter and are often used as the first line of treatment in order to supplement a deficient natural tear film to treat dryness and irritation. ${ }^{10,11}$ For a sustained therapeutic effect, these formulations should remain on the ocular surface for a sufficiently long time, but newly secreted tears can dilute and wash away active agents, and blinking can remove instilled tear substitutes, which flow through the main excretory ducts. ${ }^{10-13}$ Their relative short retention time leads to high frequency of instillation. Generally speaking, there are two major types of ATs, one that supplements the aqueous part of the tear and the other that supplements the lipid part. ${ }^{10}$ Aqueous-supplementing ATs are expected to provide lubrication and an enhancement of viscosity. ${ }^{10}$ Among them, there are simple saline-based solutions and other ATs containing natural and synthetic polymers. ${ }^{10}$ Ingredients are typically polyacrylic acid, carboxymethyl cellulose (CMC), dextran, hyaluronic acid (HA), hydroxypropyl guar, hydroxypropyl methylcellulose (HPMC), polyvinyl alcohol (PVA), polyvinylpyrrolidone (PVP), and polyethylene glycol. ${ }^{10}$ These ingredients prolong the time that the ATs stay on the eye. Specifically, HA, a long polysaccharide with a high molecular weight which is present in connective tissue and in other parts of the human body, ${ }^{14,15}$ is a component of many ATs in the form of its sodium salt (sodium hyaluronate). Due to its viscosity-enhancing properties, some manufacturers also add sodium hyaluronate directly in the solutions of the CL blister to improve the comfort after CL insertion. ${ }^{15-18}$ Lipid-supplementing ATs were introduced because a lipid deficit can cause a quicker evaporation of the tears leading to a condition of dry eye. ${ }^{10}$ These are typically emulsions where lipid droplets are suspended, such as polar phospholipid, dimyristoyl-phosphatidyl-glycerol (DMPG), propylene glycol. ${ }^{19,20}$

In the literature, several studies on the clinical effects of ATs and on the comparison between them are reported. ${ }^{13,21-29}$ Some of these studies concern the retention time on the ocular surface and both symptoms and signs in patients with dry eye. In rat and rabbit animal models of dry eye, sodium hyaluronate was reported to show a significantly longer retention time than other ATs, including carboxymethylcellulose and hydroxypropyl methylcellulose. ${ }^{13}$ Aragona et al found that sodium hyaluronate is also able to improve the conjunctival epithelial cell abnormalities of the ocular surface. ${ }^{22}$

Recent studies found that more than half of the CL wearers suffer from dry eye, a much higher percentage than found in the general population matched for age. 8,23 In CL wearers, a stable tear film is a requirement because it keeps the CL hydrated, ensures an adequate oxygen transmission, and reduces the chances of bacterial contamination of the CL. ${ }^{30-32}$ However, the interaction between tear film and CLs is certainly affected by the frequency of replacement of CLs for the different building up of deposits on the lens surface which is potentially reduced by the use of daily disposable CLs. Moreover, daily disposable CLs require the least amount of upkeep and have the advantage of reducing complications associated with case contamination and use of care products. ${ }^{33-41}$ However, the costs are typically higher and throwing the CLs every day means increased waste disposal due to the CL itself and its packaging.

The present work concerns CL wear combined with the use of ATs. The question that gave rise to this work was to investigate whether the efficacy of an aqueoussupplementing AT in symptoms and ocular signs is different in wearers of daily disposable CLS (DD-Ws), in wearers of CLs of the same material with lower frequency replacement, and in non-wearers (N-Ws).

\section{Materials and Methods}

\section{Artificial Tears}

OPTOyalA and OPTOidro ATs (OPTOX, Italy) were used in this work in single-dose format $(0.35 \mathrm{~mL}$ each). They are both isotonic saline solutions buffered to $\mathrm{pH}$ 7.2. In addition, OPTOyalA contains, as declared by the manufacturer, sodium salt of hyaluronic acid $(1.5 \mathrm{mg} / \mathrm{mL})$, L-proline $(0.752 \mathrm{mg} / \mathrm{mL})$, L-glycine $(1 \mathrm{mg} / \mathrm{mL})$, L-lysine hydrochloride $(0.14 \mathrm{mg} / \mathrm{mL})$, and L-leucine $(0.108 \mathrm{mg} / \mathrm{mL})$.

The osmolarity of the ATs was measured by the TearLab ${ }^{\mathrm{TM}}$ osmolarity system (Tear Lab, California, USA). The measured values (mean \pm standard deviation of five repetitions) were $292 \pm 2$ Osm/L (OPTOyalA) and $290 \pm 2$ Osm/L (OPTOidro).

The refractive index (mean \pm standard deviation of five repetitions) was measured (OPTOyalA: 1.33584 \pm 0.00004 ; OPTOidro: $1.33543 \pm 0.00004)$ by a digital refractometer Atago RX3000 $\alpha$ for liquids (Atago, Japan). 
The determination of relative viscosity was carried out at $18^{\circ} \mathrm{C}$ by using a homemade capillary viscometer. The relative viscosity $\left(\eta_{r}=\eta / \eta_{0}\right)$ was evaluated by correlating two measurements: one performed on the AT $(\eta)$ and the other on an isotonic solution containing $0.9 \%$ sodium chloride without preservatives (Alcon Vision Care, USA) used as reference $\left(\eta_{0}\right)$. The measured value of $\eta_{r}$ was 3.26 \pm 0.01 for OPTOyalA and $1.04 \pm 0.01$ for OPTOidro (mean \pm standard deviation of five repetitions).

\section{Contact Lenses}

Either daily disposable or 1-month manufacturerrecommended replacement CLs of the same material were included in the study. The 1-month manufacturerrecommended replacement CLs were actually used for 2 weeks (BW-W group) in the study. Some of their properties are shown in Table 1. Daily disposable CLs (somofilcon A Clarity 1 Day, Cooper Vision, USA) were used by 18 wearers (DD-Ws), as described below, while 1-month replacement CLs (somofilcon A Clariti Elite, Cooper Vision, USA) for daily use were worn by 20 wearers (BWWs). The Refine One Step hydrogen peroxide solution (Cooper Vision, USA) was also given to BW-Ws for the overnight CL storage.

\section{Participants and Study Design}

The study was conducted according to the tenets of the Declaration of Helsinki. Before being enrolled in the study each subject expressed his/her written informed consent and gave the researchers permission to collect and treat personal and optometric data. The subjects took part in the project for

Table I Contact Lenses

\begin{tabular}{|l|l|l|}
\hline Material & Somofilcon A & Somofilcon A \\
\hline Brand & Clarity I Day & Clariti Elite \\
\hline Manufacturer & $\begin{array}{l}\text { Cooper Vision, } \\
\text { USA }\end{array}$ & Cooper Vision, USA \\
\hline Replacement & Daily & $\begin{array}{l}\text { Monthly (worn for two } \\
\text { weeks in this work) }\end{array}$ \\
\hline $\begin{array}{l}\text { Equilibrium water } \\
\text { content (\%) }\end{array}$ & 56 & 56 \\
\hline $\begin{array}{l}-3.00 D \text { central } \\
\text { thickness (mm) }\end{array}$ & 0.07 & 0.07 \\
\hline $\begin{array}{l}\text { Elastic modulus } \\
\text { (MPa) }\end{array}$ & 0.50 & 0.50 \\
\hline
\end{tabular}

Note: Properties of the contact lenses used in this work. free. The Optics and Optometry Board of the University of Milano Bicocca granted approval for the study (June 2018). A scheme of the study design is shown in Figure 1.

During the recruitment phase, the inclusion criteria were the absence of any known ocular and systemic pathologies, not having used any eye drops (ATs included) in the week before the study began, and, only for nonwearers, never having worn CLs.

Thirty-three N-Ws were recruited and, on the first day (Figure 1), a standard protocol was carried out (described in the clinical assessment section below) to assess ocular symptoms and the condition of tear film and ocular surface. These subjects were randomly assigned to one of two $\mathrm{N}-\mathrm{W}$ subgroups based on the dispensed ATs (Figure 1 and Table 2): 16 subjects out of 33 were assigned at the OPTOyalA subgroup and 17 were assigned at the OPTOidro subgroup. All 33 subjects received an adequate number of packages of ATs in single-dose formats $(0.35 \mathrm{~mL}$ each) to allow using the assigned AT for 2 weeks of use (3 times a day at times chosen by each subject, each application separated from the previous one by at least 3 hours). The clinical assessment was repeated on the fifteenth day (Figure 1).

Thirty-eight CL wearers (habitual or occasional wearers of CLs of different materials and different replacement modality) were recruited and randomly assigned to one of the two groups: 18 subjects in the DD-W group and 20 subjects in the BW-W group (Figure 1). All subjects received a proper number of the assigned CLs (thirty blisters of daily disposable CLs and a pair of 1-month replacement CLs of the appropriate optical power) to be used on a daily wear basis ( $8 \pm 1$ hours a day) for 2 weeks without any AT. For the BW-W group, subjects were also provided with a package of hydrogen peroxide solution (Refine One Step, Cooper Vision, USA) as the CL care system. For CL wearers, the experimental phase of this study began at the end of these 2 weeks of preliminary wear (as shown by a dashed box in Figure 1). On the first day of the experimental phase, the standard protocol (clinical assessment) to assess symptoms, tear film, and ocular surface was carried out. Each group (DD-Ws and BW-Ws) was further divided into two subgroups depending on the randomly assigned AT (Figure 1 and Table 2). Nine out of 18 DD-Ws were included in the DD-W subgroup using OPTOyalA, the other 9 DD-Ws were included in the DD-W subgroup using OPTOidro. Eight out of $20 \mathrm{BW}-\mathrm{Ws}$ were included in the BW-W subgroup using OPTOyalA, and the other $12 \mathrm{BW}-\mathrm{Ws}$ were included in the 


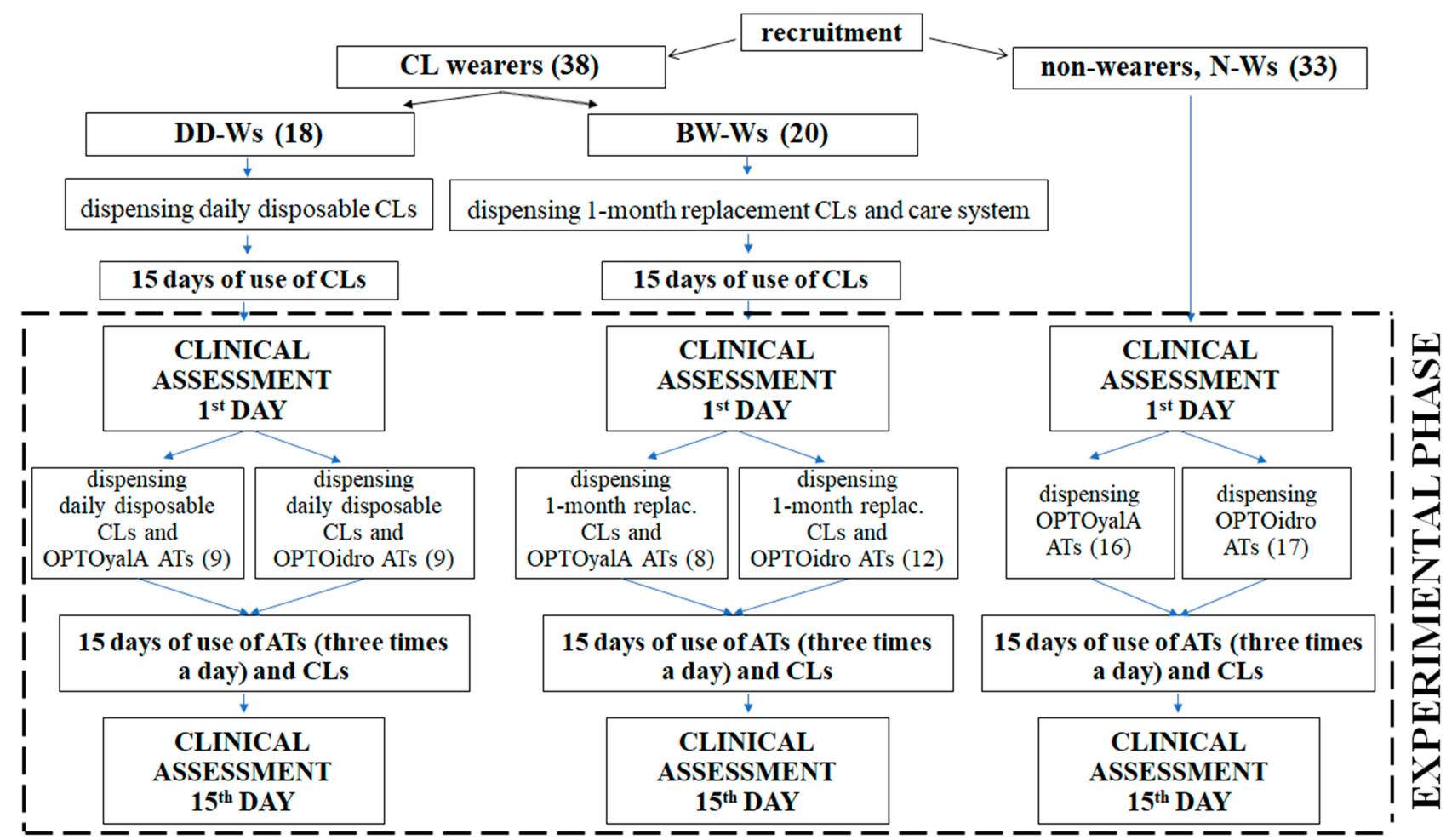

Figure I Scheme of the study design showing the recruitment of non-wearers (N-Ws) and wearers of contact lenses (CLs), either daily disposable contact lenses (DD-Ws) or biweekly replacement contact lenses (BW-Ws), and the 15-day experimental phase in which artificial tears (ATs) were used.

OPTOidro subgroup. New CLs were then provided: a pair of 1-month replacement CLs were dispensed to the group of BW-Ws to be used in the following 2 weeks together with the assigned ATs and 30 blisters of daily disposable CLs were dispensed to the DD-Ws. After 2 weeks of wear, the clinical assessment was repeated (Figure 1). CLs were worn for $8 \pm 1$ hours every day. ATs were instilled three times a day with the CL in-situ, at times chosen by each subject, each application separated from the previous one by at least 3 hours.
All participants of the three groups (N-Ws, DD-Ws, and $\mathrm{BW}-\mathrm{Ws}$ ) completed the project and stated that they had followed the instructions provided. The refractive error (spherical equivalent) of the study participants was included in the range between $-12.00 \mathrm{D}$ and +2.00 $\mathrm{D}($ mean $=3.39 \mathrm{D}$, std dev $=2.67 \mathrm{D})$.

\section{Clinical Assessment}

The clinical assessment consisted of the ocular surface symptoms measurement gathered through the use of two

Table 2 Groups and Subgroups

\begin{tabular}{|c|c|c|c|c|c|c|c|}
\hline \multirow[t]{2}{*}{ Group } & \multirow[t]{2}{*}{ Subgroup } & \multirow[t]{2}{*}{$\mathbf{N}_{\text {males }}$} & \multirow[t]{2}{*}{$\mathbf{N}_{\text {females }}$} & \multirow[t]{2}{*}{$N_{\text {subgroup }}$} & \multirow[t]{2}{*}{$\mathbf{N}_{\text {group }}$} & \multicolumn{2}{|c|}{ Age (years) } \\
\hline & & & & & & Mean & Std Dev \\
\hline $\mathrm{N}-\mathrm{W}$ & $\begin{array}{l}\text { OPTOyalA } \\
\text { OPTOidro }\end{array}$ & $\begin{array}{l}10 \\
7\end{array}$ & $\begin{array}{l}6 \\
10\end{array}$ & $\begin{array}{l}16 \\
17\end{array}$ & 33 & $\begin{array}{l}25.1 \\
23.2\end{array}$ & $\begin{array}{l}3.2 \\
2.7\end{array}$ \\
\hline DD-W & $\begin{array}{l}\text { OPTOyalA } \\
\text { OPTOidro }\end{array}$ & $\begin{array}{l}3 \\
5\end{array}$ & $\begin{array}{l}6 \\
4\end{array}$ & $\begin{array}{l}9 \\
9\end{array}$ & 18 & $\begin{array}{l}22.0 \\
22.9\end{array}$ & $\begin{array}{l}1.3 \\
1.1\end{array}$ \\
\hline BW-W & $\begin{array}{l}\text { OPTOyalA } \\
\text { OPTOidro }\end{array}$ & $\begin{array}{l}4 \\
4\end{array}$ & $\begin{array}{l}4 \\
8\end{array}$ & $\begin{array}{l}8 \\
12\end{array}$ & 20 & $\begin{array}{l}23.5 \\
25.0\end{array}$ & $\begin{array}{l}1.2 \\
2.3\end{array}$ \\
\hline
\end{tabular}

Notes: Number of males/females, total number of subjects $\left(\mathrm{N}_{\text {subgroup }}\right)$, mean age, and standard deviation of the age for each subgroup (OPTOyalA and OPTOidro) of the three groups (non-wearers, wearers of daily disposable contact lenses, wearers of biweekly replacement contact lenses).

Abbreviations: $\mathrm{N}_{\text {males }}$, number of males; $\mathrm{N}_{\text {females }}$, number of females; $\mathrm{N}_{\text {subgroup }}$, total number of subjects of the subgroup; $\mathrm{N}_{\text {group }}$, total number of subjects of the group; $\mathrm{N}-\mathrm{W}$, non-wearers; DD-W, wearers of daily disposable contact lenses; BW-W, wearers of biweekly replacement contact lenses. 
standardized questionnaire and in three objective measurements of tear film and ocular surface: tear meniscus height (TMH), non-invasive tear break-up time (NIBUT), and ocular redness evaluation (OR).

The two questionnaires to evaluate ocular surface symptoms were the Ocular Surface Disease Index (OSDI) ${ }^{42}$ and the 5-Item Dry Eye Questionnaire (DEQ-5). ${ }^{43}$

The TMH measurement, performed to achieve a measure of tear volume, ${ }^{44}$ was carried by a two-step procedure as described by the Dry Eye Report (CSO, Firenze, Italy) integrated in the software platform called Phoenix (CSO, Firenze, Italy): a digital image of the inferior tear meniscus was firstly acquired, then the height of the meniscus in a central position (just under the virtual extension of the vertical axes of the cornea) was measured by the digital ruler device of the software. The evaluation was repeated three times and the mean value was taken into consideration

The non-invasive tear break-up time (NIBUT) was performed using the Dry Eye Report (DER) (CSO, Firenze, Italy). The software allowed to detect the first tear break up through an automatic algorithm. The measurement was repeated three times and the mean value was taken into consideration.

OR was measured because considered the most common clinical sign that is suggestive of ocular surface inflammation. ${ }^{44}$ A snapshot of temporal bulbar conjunctiva was taken by the Dry Eye Report (CSO, Firenze, Italy) and the bulbar ocular redness. All images were saved as masked codes and OR severity was subsequently determined by comparison with the templates of Efron Grading scales. $^{45}$

In the clinical assessment sequence, each subject was firstly requested to complete the two questionnaires then the objective assessment was carried out in the following order: TMH, NIBUT, and OR. The two repeated clinical assessments were carried out at the same time in the morning (with a tolerance of 1 hour) in order to minimize possible effect of diurnal variation. ${ }^{46}$ For each subject, only the data of the right eye were analyzed.

\section{Statistical Analysis}

Descriptive statistics (mean, standard deviation SD) was produced for the measurements (OSDI, DEQ5, TMH, NIBUT, OR) collected on the first day and on the fifteenth day of the experimental phase. For each subject, the percentage change $(\Delta)$ of each variable was also calculated as the difference between the values measured on the fifteenth day and on the first day divided by the value of the first day. Mean and SD were also calculated for these percentage changes.

For each group separately (N-Ws, DD-Ws, BW-Ws), a preliminary analysis was performed to compare the results of the two subgroups based on the type of AT (OPTOyalA or OPTOidro). Due to the small number of subjects, non-parametric statistics were used (unpaired Mann-Whitney, level of significance: p-value $<0.05$ ). The subsequent analyses concerned (i) the three groups consisting of all N-Ws, all DD-Ws, and all BW-Ws regardless of the assigned AT, (ii) the three groups consisting of N-Ws using OPTOyalA, DD-Ws using OPTOyalA, and BW-Ws using OPTOyalA, and finally (iii) the three groups consisting of N-Ws using OPTOidro, DD-Ws using OPTOidro, and BW-Ws using OPTOidro. The comparison between the results obtained in each group on the first day and on the fifteenth day was performed by the non-parametric Wilcoxon test (level of significance: $\mathrm{p}$-value $<0.05$ ).

\section{Results and Discussion}

For each group separately (N-Ws, DD-Ws, BW-Ws) and for each subgroup (OPTOidro and OPTOyalA), Figure 2 shows the mean values measured on the 1 st and 15th days. The values are also tabulated in Table 3, together with the mean percentage variations $(\Delta)$ for each variable, which allow to probe the effect of the ATs without the subjects' initial individual variability influencing the data analysis.

A preliminary analysis was carried out to compare the results of the two subgroups. The p-values obtained by unpaired comparison between the $\Delta$ values of the two subgroups (OPTOyalA vs OPTOidro) are also tabulated in Table 3 for each group. Only for the TMH of BW-Ws (1 comparison out of 15), the effect of the two ATs was noted to be different ( $\mathrm{p}=0.041$ in Table 3$)$. However, neither of the two corresponding TMH $\Delta$ values showed a significant variation between the 1 st and the 15 th day $(+14 \%$ in the case of OPTOyalA showing $\mathrm{p}=0.263$ and $-12 \%$ in the case of OPTOidro showing $\mathrm{p}=0.195$ ). This makes the difference between the two ATs in the unpaired comparison of little clinical significance. No significant differences were noted in the unpaired comparison between the two subgroups (OPTOyalA vs OPTOidro, $\mathrm{p}>0.05$ ) for all other variables. Looking carefully at Table 3, some other apparent discrepancies concern OR of N-Ws, DEQ5 of DD-Ws, and NIBUT of BW-Ws. Concerning the OR of N-Ws, the variation between the 

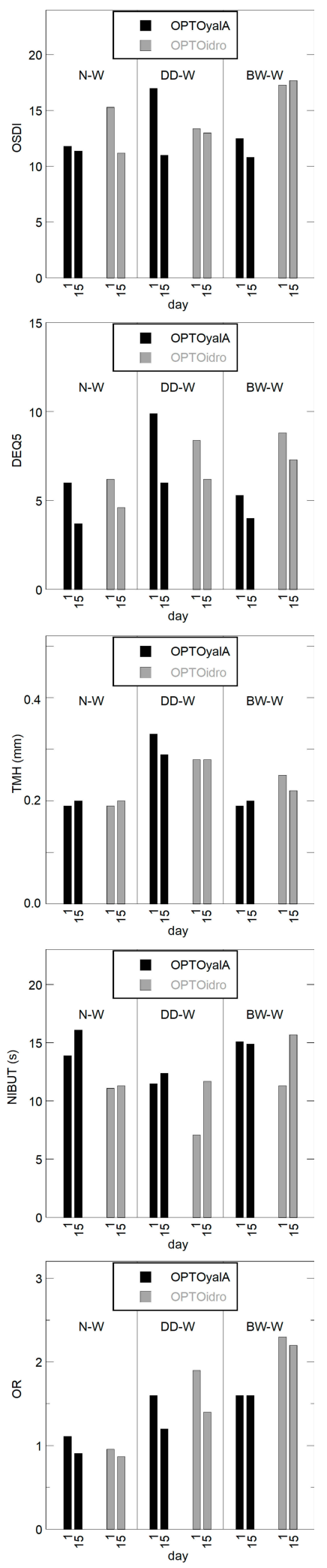

Figure 2 Mean data measured on the Ist and I5th days for the two ATs separately. Abbreviations: N-Ws, non-wearers; DD-Ws, wearers of daily disposable contact lenses; BW-Ws, wearers of biweekly replacement contact lenses; OSDI, Ocular Surface Disease Index; DEQ5, 5-Item Dry Eye Questionnaire; TMH, tear meniscus height; NIBUT, non-invasive tear break-up time; OR, ocular redness.
1 st and the 15th day was significant only for the OPTOyalA subgroup and for the whole group of 33 subjects. Although it was not significant for the OPTOidro subgroup ( $\mathrm{p}=0.130$ ), the overall result of all the $33 \mathrm{~N}-\mathrm{Ws}$ well represents this group. Indeed, all three OR $\Delta$ values of N-Ws are negative $(-10 \%,-12 \%,-8 \%)$ and the unpaired comparison between OPTOyalA and OPTOidro did not result in a significant difference $(p=0.416)$. The scenario is similar for the DEQ5 of DD-Ws. All three DEQ5 $\Delta$ values of DD-Ws are negative $(-34 \%,-44 \%$, $-24 \%$ ) and the comparison between OPTOyalA and OPTOidro did not result in a significant difference $(\mathrm{p}=0.352)$. Although the variation between the $1 \mathrm{st}$ and the 15th day was not significant for the OPTOidro subgroup ( $\mathrm{p}=0.175$ ), the overall result of all the 18 DD-Ws well represents the DD-W group. Finally, concerning the NIBUT of BW-Ws, the variation between the 1st and the 15th day was significant only for the OPTOidro subgroup and for the whole group of 33 subjects. Although it was not significant for the OPTOyalA subgroup $(\mathrm{p}=0.799)$, the overall result of all the $20 \mathrm{BW}-\mathrm{Ws}$ well represents this group. Indeed, all three $\Delta$ values are positive $(+35 \%$, $+29 \%,+40 \%)$ and the comparison between OPTOyalA and OPTOidro did not result in a significant difference $(p=0.160)$. In all the other 11 out of 15 cases of Table 3, the variations after 15 days of the OPTOyalA subgroup only, of the OPTOidro subgroup only, and of the group as a whole showed the same type of behavior and the same statistical evidence. Based on these considerations, the subsequent analyses concerned the three groups consisting of $33 \mathrm{~N}-\mathrm{Ws}, 18 \mathrm{DD}-\mathrm{Ws}$, and $20 \mathrm{BW}-\mathrm{Ws}$ regardless of the assigned AT.

Even if the purpose of this work was the comparison between the 1st and the 15th day, a first comment concerns the data obtained on the 1st day in the three groups. The results are compatible with the expected results for young and healthy subjects. The most interesting parameter was found to be the OR because a statistically greater average value was found for the $18 \mathrm{DD}-\mathrm{Ws}$ on the 1st day than for the $33 \mathrm{~N}$-Ws $(1.7 \pm 0.3$ vs $1.0 \pm 0.6)$, as well as for the 20 BW-Ws compared to N-Ws $(2.0 \pm 0.6$ vs $1.0 \pm 0.6)$. Even if it is not reported in Table 3, an unpaired MannWhitney test between the data taken on the 1st day provided $\mathrm{p}<0.001$ in both of these comparisons. After 15 days, a significant OR improvement was found in N-Ws $(-10 \%)$ and DD-Ws (-23\%). From a clinical point 


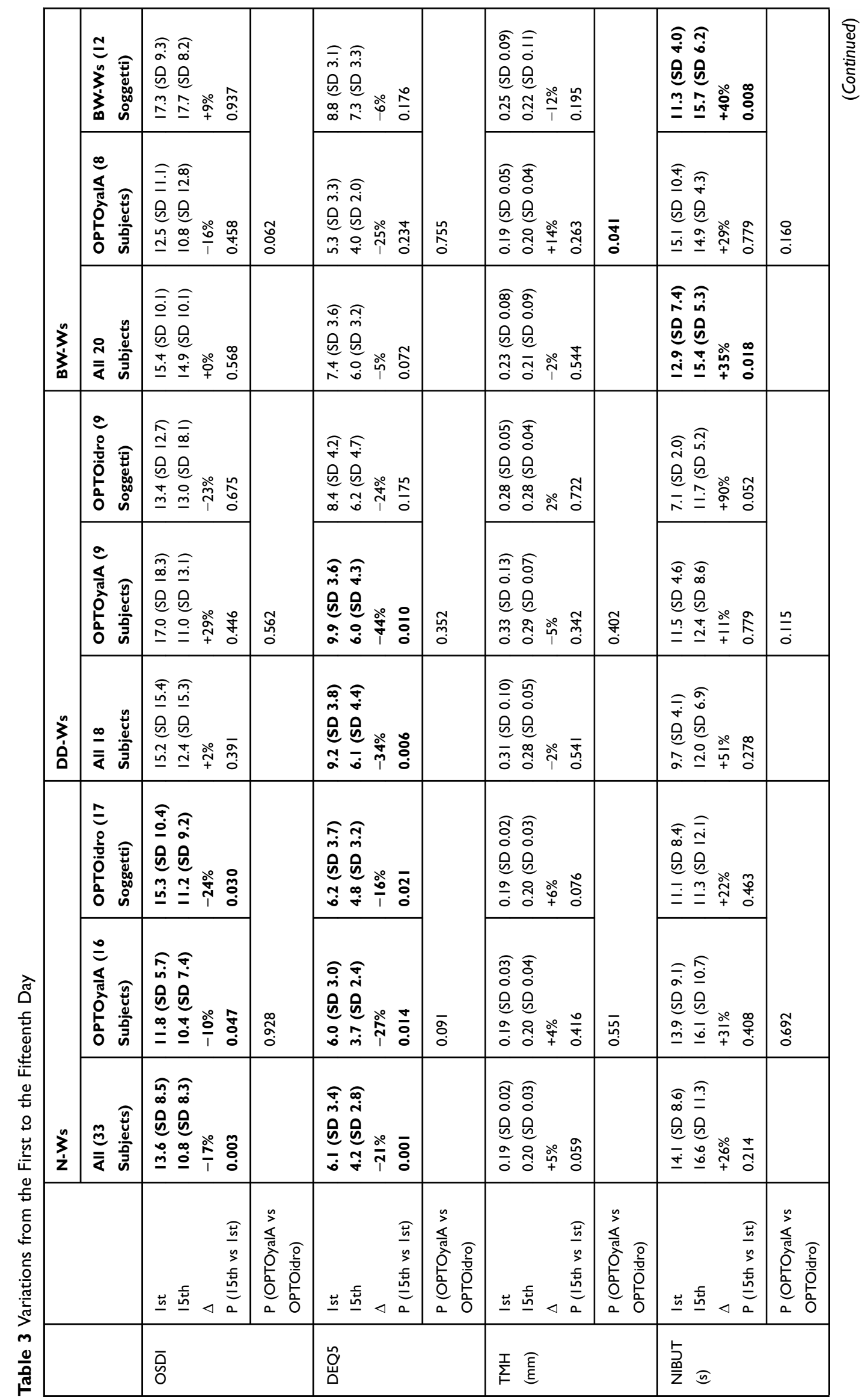




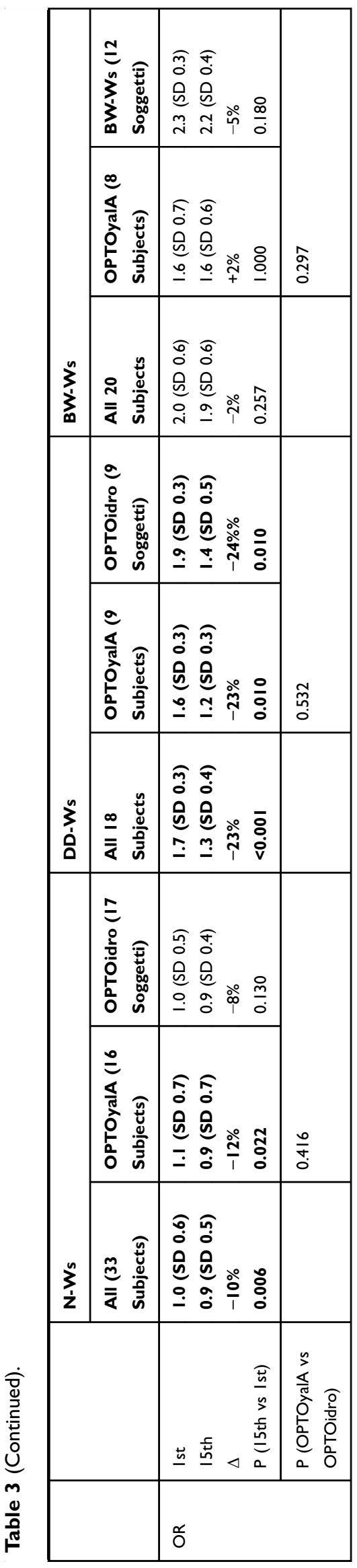

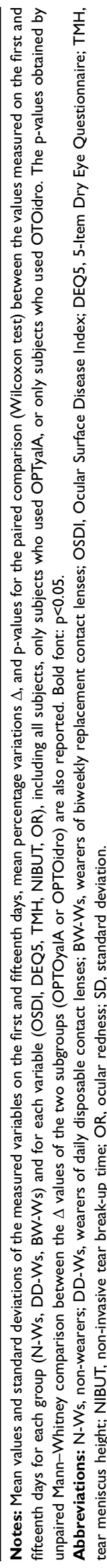

of view, the initial condition of N-Ws was already that of healthy and young subjects and the AT effect, although statistically significant, is clinically less important. More interesting is the significant AT effect on the OR of DDWs, considering their initial worse condition. The same cannot be said for the OR of BW-Ws. Their initial condition was also worse than in N-Ws, but no significant improvement was seen with the use of ATs.

The other parameter that showed a statistically significant variation in DD-Ws was DEQ-5 (-34\%). Also in this case, the improvement was not significant in BW-Ws, while it was significant for $\mathrm{N}-\mathrm{Ws}(-21 \%)$, but less relevant from the clinical point of view, their initial condition being already fully compatible with that of young and healthy subjects. For all three groups, no significant variation was found for tear volume (TMH). Concerning tear stability, the mean NIBUT was higher after 15 days in all 3 groups $(+26 \%,+51 \%$, and $+35 \%)$, but the difference was statistically significant only for BW-Ws.

As such, the main finding of this study is the general significant improvement of the external eye comfort and condition (DEQ5 and OR in Table 3) evaluated after 15 days of use of the ATs under investigation in N-Ws and in DD-Ws, without any significant change of the TMH (Table 3). This indicates that the aqueous-supplementing effect of the AT is more a rinsing and tear replacement effect than an increase in tear volume. Not having found significant differences between the two ATs (Table 3 and mean data shown in Figure 2) represents a further confirmation of a positive effect mainly due to simple rinsing and tear replacement, as OPTOidro is a simple saline solution. This work concerns young and healthy subjects. In other studies reported in the literature, differences between different ATs were found when applied to dry eye subjects. The highest viscosity ATs were found to be preferable to treat these cases. ${ }^{12,13,22,27}$ In patients with deficient lipid layers, lipid emulsion eyedrops were also found to produce positive effects. ${ }^{23,25}$

Rinsing and replacement of tear film were not enough to produce any clear and statistically significant OR and comfort benefit in the case of 1-month replacement CLs of the same material used during the day for $8 \pm 1$ hours a day for 2 weeks (BW-Ws). This can be due to various reasons. It must be said that the care system used in this work is the same as that used in a previous study ${ }^{29}$ in which monthly comfilcon A silicone-hydrogels CLs were used for 15 days. In that study, the external-eye condition of CL wearers was evaluated before and after 15 days of daily 
wear combined with different care systems: hydrogen peroxide such as in the present work (first group in Moro et $\mathrm{al}^{29}$ ), detergent solution combined with hydrogen peroxide (second group in Moro et $\mathrm{al}^{29}$ ), and multipurpose solution (third group in Moro et $\mathrm{al}^{29}$ ). That study was aimed at comparing the different maintenance systems. A worsening of the external-eye condition was reported only in the first group, which can be attributed to a lower cleaning efficacy of the hydrogen peroxide compared to the other two care systems or to a different effect on the CL wettability, surface friction, or on other CL properties. The present study highlights that the use of the ATs under investigation at least prevents the worsening after 2 weeks of wear of the monthly CLs treated with hydrogen peroxide. Unfortunately, ATs did not significantly improve the symptoms and ocular signs in BW-Ws.

An extension of this work could be the study of the effects of OPTOyalA and OPTOidro ATs in wearers of other types of CLs of different materials, also with the aim to shed more light on the reason why the effectiveness of ATs was not found in the case of BW-Ws. The study could also include chemical-physical analysis of the CLs used together with the ATs, eg, surface wettability and friction measurements, ${ }^{47,48}$ surface morphology and elemental characterization. ${ }^{49,50}$

\section{Conclusions}

On young and healthy CL wearers, the aqueoussupplementing effect of the ATs under consideration was mainly a rinsing and tear replacement effect, which allowed an improvement of the ocular redness and eye comfort, without any significant increase in tear volume after 2 weeks of use. The efficacy of the ATs appeared to be correlated with the replacement schedule of the lenses. There was a less significant improvement in monthly replacement CL wearers compared with daily disposable CL wearers. Nonetheless, ATs appeared to prevent the worsening of the external eye condition that was previously reported after 2 weeks of silicone-hydrogel CL wear combined with hydrogen peroxide cleaning system. The efficacy of the ATs was observed also in the control group (non-wearers), although it is less relevant from the clinical point of view, given their initial condition of young and healthy subjects.

\section{Disclosure}

The authors report no conflicts of interest in this work.

\section{References}

1. Peters E, Colby K. Chapter 3: the tear film. In: Tasman W, Jaeger EA, editors. Duane's Ophthalmology. Philadelphia: Lippincott Williams and Wilkins; 2013. Available from: https://ophed.net/system/files/ 2009/07/tear-film-duanes-ophthalmology-1768-1768.pdf.

2. Dart DA, Willcox MDP. Complexity of the tear film: importance in homeostasis and dysfunction during disease. Exp Eye Res. 2013;117:1-3. doi:10.1016/j.exer.2013.10.008

3. Hodges RR, Dartt DA. Tear film mucins: front line defenders of the ocular surface; comparison with airway and gastrointestinal tract mucins. Exp Eye Res. 2013;117:62-78. doi:10.1016/j.exer.2013.07.027

4. Bron AJ, Tiffany JM, Gouveia SM, Yokoi N, Voon LW. Functional aspects of the tear film lipid layer. Exp Eye Res. 2004;78:347. doi:10.1016/j.exer.2003.09.019

5. Johnson ME, Murphy AJP. Changes in the tear film and ocular surface from dry eye syndrome. Progr Ret Eye Res. 2004;23:449-474. doi:10.1016/j.preteyeres.2004.04.003

6. Gayton JL. Etiology, prevalence, and treatment of dry eye disease. Clin Ophthalmol. 2009;3:405-412. doi:10.2147/OPTH.S5555

7. Wolkoff P. Ocular discomfort by environmental and personal risk factors altering the precorneal tear film. Toxicol Lett. 2010;199:203-212. doi:10.1016/j.toxlet.2010.09.001

8. Stapleton F, Alves M, Bunya VY, et al. Tfos dews ii epidemiology report. Ocul Surf. 2017;15:334-365. doi:10.1016/j.jtos.2017.05.003

9. Zhang X, Vj M, Qu Y, et al. Dry eye management: targeting the ocular surface microenvironment. Int J Mol Sci. 2017;18:E1398. doi:10.3390/ijms 18071398

10. Jones L, Downie LE, Korb D, et al. TFOS DEWS II management and therapy report. Ocul Surf. 2017;15:575-628. doi:10.1016/j.jtos.2017.05.006

11. Pastori V, Tavazzi S, Lecchi M. Lactoferrin-loaded contact lenses: eye protection against oxidative stress. Cornea. 2015;34:693-697. doi:10.1097/ICO.0000000000000435

12. Hall JQ Jr, Ridder WH 3rd, Nguyen AL, Paugh JR. Visual effect and residence time of artificial tears in dry eye subjects. Optom Vis Sci. 2011;88:872-880. doi:10.1097/OPX.0b013e31821b0b2c

13. Zheng X, Goto T, Ohashi Y. Comparison of in vivo efficacy of different ocular lubricants in dry eye animal models. Invest Ophthalmol Vis Sci. 2014;55:3454-3460. doi:10.1167/iovs.13-13730

14. Kobayashi Y, Okamoto A, Nishinari K. Viscoelasticity of hyaluronic acid with different molecular weights. Biorheol. 1994;31:235-244. doi:10.3233/BIR-1994-31302

15. Rah M. Hyaluronan: a review of its properties, ophthalmic uses and research. Cont Lens Spectr. 2010.

16. Fagnola M, Pagani MP, Maffioletti S, Tavazzi S, Papagni A. Hyaluronic acid in hydrophilic contact lenses: spectroscopic investigation of the content and release in solution. Cont Lens Anter Eye. 2009;32:108-112. doi:10.1016/j.clae.2009.02.006

17. Tavazzi S, Ferraro L, Fagnola M, et al. Mechanically triggered solute uptake in soft contact lenses. Colloids Surf B Biointerfaces. 2015;130:16. doi:10.1016/j.colsurfb.2015.03.057

18. Tavazzi S, Tonveronachi M, Fagnola M, et al. Wear effects on microscopic morphology and hyaluronan uptake in siloxane-hydrogel contact lenses. J Biomed Mater Res B Appl Biomater. 2015;103(5):1092. doi:10.1002/jbm. b. 33278

19. Benelli U. Systane ${ }^{\circledR}$ lubricant eye drops in the management of ocular dryness. Clin Ophthalmol. 2011;5:783-790. doi:10.2147/OPTH.S13773

20. Garrigue JS, Amrane M, Faure MO, Holopainen JM, Tong L. Relevance of lipid-based products in the management of dry eye disease. $J$ Ocul Pharmacol Ther. 2017;33:647-661. doi:10.1089/jop.2017.0052

21. Hamano T, Horimoto K, Lee M, Komemushi S. Sodium hyaluronate eye drops enhance tear film stability. Jpn J Ophthalmol. 1996;40:62-65.

22. Aragona P, Papa V, Micali A, Santocono M, Milazzo G. Long term treatment with sodium hyaluronate-containing artificial tears reduces ocular surface damage in patients with dry eye. $\mathrm{Br} \mathrm{J} \mathrm{Ophthalmol.}$ 2002;86:181-184. doi:10.1136/bjo.86.2.181 
23. Scaffidi RC, Korb DR. Comparison of the efficacy of two lipid emulsion eye drops in increasing tear film lipid layer thickness. Eye Contact Lens. 2007;33:38-44. doi:10.1097/01.icl.0000247638.50568.c0

24. Calvao-Santos G, Borges C, Nunes S, Salgado-Borges J, Duarte L. Efficacy of 3 difference artificial tears for the treatment of dry eye in frequent computer users and/or contact lens users. Eur J Ophthalmol. 2011;21:538-544. doi:10.5301/EJO.2011.6324

25. Lee SY, Tong L. Lipid-containing lubricants for dry eye: a systematic review. Optom Vis Sci. 2012;89:1654-1661. doi:10.1097/OPX.0b0 $13 \mathrm{e} 31826 \mathrm{f} 32 \mathrm{e} 0$

26. Daull P, Lallemand F, Garrigue JS. Benefits of cetalkonium chloride cationic oil-in-water nanoemulsions for topical ophthalmic drug delivery. J Pharm Pharmacol. 2014;664:531-541. doi:10.1111/ jphp. 12075

27. Williams DL, Mann BK. Efficacy of a crosslinked hyaluronic acid-based hydrogel as a tear film supplement: a masked, controlled study. PLoS One. 2014;9:e99766. doi:10.1371/journal.pone.0099766

28. Torkildsen G, Brujic M, Cooper MS, et al. Evaluation of a new artificial tear formulation for the management of tear film stability and visual function in patients with dry eye. Clin Ophthalmol. 2017;11:1883-1889. doi:10.2147/OPTH.S144369

29. Moro A, Fagnola M, Picarazzi S, et al. Hydrogen-peroxide and silicone-hydrogel contact lenses: worsening of external eye condition and tear film instability. Cont Lens Anterior Eye. 2018;41:157. doi:10.1016/j.clae.2017.10.008

30. Nichols JJ, Sinnott LT. Tear film, contact lens, and patient-related factors associated with contact lens-related dry eye. Invest Ophthalmol Vis Sci. 2006;47:1319-1328. doi:10.1167/iovs.05-1392

31. Downie LE, Craig JP. Tear film evaluation and management in soft contact lens wear: a systematic approach. Clin Exp Optom. 2017;100:438-458. doi:10.1111/cxo.12597

32. Picarazzi S, Lecchi M, Pastori V, D’Arienzo M, Scotti R, Tavazzi S. Photon correlation spectroscopy applied to tear analysis. Colloids Surf B Biointerfaces. 2017;157:26-30. doi:10.1016/j.colsurfb. 2017.05.057

33. Radford C, Minassian D, Dart J. Disposable contact lens use as a risk factor for microbial keratitis. $B r \quad J$ Ophthalmol. 1998;82 (11):1272-1275. doi:10.1136/bjo.82.11.1272

34. Papas EB, Carnt N, Willcox MD, Holden BA. Complications associated with care product use during silicone daily wear of hydrogel contact lens. Eye Contact Lens. 2007;33:392-393. doi:10.1097/ ICL.0b013e318157e542

35. Dart JK, Radford CF, Minassian D, Verma S, Stapleton F. Risk factors for microbial keratitis with contemporary contact lenses: a case-control study. Ophthalmol. 2008;115:1647-1654, 1654 e1-3. doi:10.1016/j.ophtha.2008.05.003

36. Zeri F, Durban JJ, Hidalgo F, Gispets J. Attitudes towards contact lenses: a comparative study of teenagers and their parents. Cont Lens Anterior Eye. 2010;33(3):119-123. doi:10.1016/j.clae.2009.12.009
37. Efron N, Efron SE, Morgan P, Morgan S. A 'cost-per-wear' model based on contact lens replacement frequency. Clin Exp Optom. 2010;93(4):253-260. doi:10.1111/j.1444-0938.2010.00488.x

38. Willcox MD, Carnt N, Diec J, et al. Contact lens case contamination during daily wear of silicone hydrogels. Optom Vis Sci. 2010;87:456-464. doi:10.1097/OPX.0b013e3181e19eda

39. Wu VT, Willcox M, Zhu H, Stapleton F. Contact lens hygiene compliance and lens case contamination: a review. Cont Lens Anterior Eye. 2015;38:307-316. doi:10.1016/j.clae.2015.04.007

40. Livi S, Zeri F, Baroni R. Health beliefs affect the correct replacement of daily-disposable contact lenses: predicting compliance with the health belief model and the theory of planned behaviour. Cont Lens Anterior Eye. 2017;40:25-32. doi:10.1016/j.clae.2016.09.003

41. Picarazzi S, Bergamaschi D, Tavazzi S. Differences between tears of contact lens wearers studied by photon correlation spectroscopy. Cont Lens Anterior Eye. 2019;42(2):212. doi:10.1016/j.clae.2018.10.004

42. Schiffman RM, Christianson MD, Jacobsen G, Hirsch JD, Reis BL. Reliability and validity of the ocular surface disease index. Arch Ophthalmol. 2000;118:615e21. doi:10.1001/archopht.118.5.615

43. Chalmers RL, Begley CG, Caffery B. Validation of the 5-item dry eye questionnaire (DEQ-5): discrimination across self-assessed severity and aqueous tear deficient dry eye diagnoses. Cont Lens Anterior Eye. 2010;33:55e60. doi:10.1016/j.clae.2009.12.010

44. Wolffsohn JS, Arita R, Chalmers R, et al. TFOS DEWS II diagnostic methodology report. Ocul Surf. 2017;15:539-574. doi:10.1016/j. jtos.2017.05.001

45. Scale EG, Efron N. Contact Lens Complications. Oxford: Butterworth-Heinemann; 1999.

46. Shen M, Wang J, Tao A, et al. Diurnal variation of upper and lower tear menisci. Am J Ophthalmol. 2008;145:801-806. doi:10.1016/j. ajo.2007.12.024

47. Sterner O, Aeschlimann R, Zürcher S, et al. Friction measurements on contact lenses in a physiologically relevant environment: effect of testing conditions on friction. Invest Ophthalmol Vis Sci. 2016;57:5383-5392. doi:10.1167/iovs.16-19713

48. Lin MC, Svitova TF. Contact lenses wettability in vitro: effect of surface-active ingredients. Optom Vis Sci. 2010;87:440-447. doi:10.1097/OPX.0b013e3181dc9a1a

49. Bettuelli M, Trabattoni S, Fagnola M, Tavazzi S, Introzzi L, Farris S. Surface properties and wear performances of siloxane-hydrogel contact lenses. J Biomed Mater Res B Appl Biomater. 2013;101:1585-1593. doi:10.1002/jbm.b.32901

50. Zeri F, Borghesi A, Acciarri M, Tavazzi S. Interaction between siloxane-hydrogel contact lenses and eye cosmetics: aluminium as a marker of adsorbed mascara deposits. Polym Polym Comp. 2020. doi:10.1177/0967391120922421
Clinical Optometry

\section{Publish your work in this journal}

Clinical Optometry is an international, peer-reviewed, open access journal publishing original research, basic science, clinical and epidemiological studies, reviews and evaluations on clinical optometry. All aspects of patient care are addressed within the journal as well as the practice of optometry including economic and business analyses. Basic and clinical research papers are published that cover

Submit your manuscript here: https://www.dovepress.com/clinical-optometry-journal all aspects of optics, refraction and its application to the theory and practice of optometry. The manuscript management system is completely online and includes a very quick and fair peer-review system, which is all easy to use. Visit http://www.dovepress.com/ testimonials.php to read real quotes from published authors. 